\title{
Wouter DRUWÉ, Scandalum in the Early Bolognese \\ Decretistic and in Papal Decretals (ca. 1140-1234)
}

\section{Arnaud Fossier}

\section{OpenEdition}

\section{Journals}

Édition électronique

URL : https://journals.openedition.org/ccm/8778

DOI : $10.4000 / \mathrm{ccm} .8778$

ISSN : 2119-1026

\section{Éditeur}

Centre d'études supérieures de civilisation médiévale/Université de Poitiers

\section{Édition imprimée}

Date de publication : 1 décembre 2021

Pagination : 391-392

ISBN : 978-2-490783-11-3

ISSN : 0007-9731

\section{Référence électronique}

Arnaud Fossier, «Wouter DRUwÉ, Scandalum in the Early Bolognese Decretistic and in Papal Decretals (ca. 1740-1234)», Cahiers de civilisation médiévale [En ligne], 256 | 2021, mis en ligne le 01 décembre 2021, consulté le 25 mai 2022. URL : http://journals.openedition.org/ccm/8778 ; DOI : https://doi.org/ $10.4000 / \mathrm{ccm} .8778$

\section{(c) (i) (9)}

La revue Cahiers de civilisation médiévale est mise à disposition selon les termes de la Licence Creative Commons Attribution - Pas d'Utilisation Commerciale - Pas de Modification 4.0 International. 
Wouter DRuwé, Scandalum in the Early Bolognese Decretistic and in Papal Decretals (ca. 1140-1234), Louvain, Peeters (Studia breviora, 1), 2018.

Le sujet de cette brève étude d'histoire du droit, tirée d'un mémoire de master soutenu à la Faculty of Canon Law de Louvain, aurait peut-être dérouté il y a quinze ans. Mais les travaux sur la notion de « scandale », ses usages, sa signification et sa portée se sont multipliés depuis, en France notamment. Qu'apporte donc ce petit opus à la littérature existante? L'inventaire méthodique qui manquait jusqu'ici, de toutes les occurrences du terme et de ses dérivés dans une partie de la littérature canonique de l'âge « classique » (1140-1234), mais aussi une relecture de certains textes souvent plus fine que celle de ses prédécesseurs - Wouter Druwé n'hésite pas, du reste, à engager le débat avec eux (avec Arnaud Fossier, p. 17; p. 19, n. 33 et p. 38, n. 28; avec Capucine Nemo-Pekelman, p. 49, n. 74; ou bien encore avec Corinne Leveleux, p. 68, n. 73). L'auteur insiste en outre sur une phase d'élaboration du droit canonique jusqu'ici négligée, en tout cas moins traitée, qui correspond à la cinquantaine d'années séparant les débuts de la composition du Décret de Gratien (v. 1130) et la fin de la première génération des décrétistes formés à Bologne (v. 1180). Le choix de s'arrêter là et d'exclure la deuxième génération des commentateurs du Décret, n'est d'ailleurs pas très bien justifié, d'autant que l'auteur prolonge ensuite son étude avec les décrétales - ces lettres des papes qui répondaient à une consultation, un appel ou une plainte - ayant été compilées dans le Liber Extra en 1234.

Tout au long de ce parcours dans le droit canonique, scandé par quatre chapitres de tailles inégales (Gratien; les décrétistes bolonais; les décrétales d'Alexandre III [1159-1181] à Grégoire IX [1227-1241]; puis la Summa de paenitentia de Raymond de Peñafort rédigée entre 1224 et 1234), l'auteur prend soin de résumer chacune de ses analyses en fin de chapitre ou de partie. Cela est d'autant plus utile que le droit ici étudié ressort comme un champ sinon de controverses, du moins d'opinions parfois très différentes. Pour Gratien par ex., le concept de scandale - qui ne concerne pas que les clercs, mais touche aussi à certaines questions afférentes aux laïcs - semble aller de soi et ne nécessite pas d'être défini. Le père de la science canonique affirme donc à de multiples reprises que le scandale doit être « évité », mais... ne dit jamais pourquoi. Rufin, qui achève sa Summa v. 1164, marque une étape importante, non seulement parce qu'il donne au mot le sens plus clair de « dispute » ou « conflit », mais aussi parce qu'il envisage toutes les mesures qui peuvent être prises face au scandale : dispense, purgation canonique, mesures disciplinaires, ou « politique du pardon » (p. 37). Pour Étienne de Tournai en revanche, une peine doit être appliquée dès lors que le scandale met en danger le salut de l'âme et il n'est alors plus question de tolérer certaines pratiques illicites. Simon de Bisignano, enfin, est le premier à lier explicitement le maintien de la paix dans l'Église et l'évitement du scandale. Globalement, donc, tous les décrétistes semblent d'accord pour faire de l'évitement du scandale une priorité, mais aucun n'en produit une théorie.

À vrai dire, les 67 décrétales du Liber Extra qui contiennent le mot «scandale » ou ses dérivés ne le théorisent pas davantage, mais le sens d' " indignation »- des fidèles ou de la hiérarchie ecclésiastique - se stabilise pour de bon. Les solutions préconisées pour y faire face restent diverses : arbitrage plutôt que dispense chez Alexandre III, purgation canonique chez Lucius III (1181-1185), procédure pénale pour Innocent III (1198-1216) - lorsque le crime commis par un clerc a suscité une rumeur d'infamie et nécessite d'être puni au plus vite. Mais il arrive aussi, pour Innocent III, qu'un scandale puisse être éteint par un simple serment purgatoire; et s'il n'y a pas de scandale, la clémence est de rigueur et une simple pénitence doit suffire. Cela prouve combien le concept est ambivalent aux yeux du souverain pontife et requiert discernement et doigté, car, selon les contextes, le scandale peut être utile ou au contraire néfaste.

Si Innocent III est à l'honneur dans ce chapitre, avec 27 décrétales usant du terme scandalum, c'est 
aussi parce qu'avec lui, le scandale devient une « technique argumentative » (p. 64), permettant à ceux qui s'adressent à lui d'attirer son attention sur une situation particulière et d'en souligner la gravité. On a en revanche plus de mal à suivre le raisonnement de l'auteur lorsqu'il affirme que, pour Innocent III, le scandale n'est pas qu'un problème lié aux crimes « manifestes », mais peut aussi avoir des conséquences au for interne (p. 70). Il n'est d'ailleurs pas anodin qu'aucun des travaux fondamentaux sur la question des fors et leur articulation (Stephan Kuttner, Paolo Prodi, Jacques Chiffoleau, ou plus récemment Wolfgang Müller) ne soit cité. Cela révèle certes que les problématiques, les références et les méthodes des historiens du droit ne sont pas toujours les mêmes que celles des historiens généralistes, mais aussi que l'un des enjeux d'une étude complète de la notion de scandale dans la tradition canonique devrait être de voir ce qu'elle dit de la place du secret dans le gouvernement ecclésial et de ce nouvel espace juridictionnel - le for interne - qu'ouvrent les canonistes et les théologiens au tournant du XII ${ }^{\mathrm{e}}$ et du XIII ${ }^{\mathrm{e}} \mathrm{s}$.

Le problème plus largement de cette étude est qu'elle ne rend pas compte de l'inscription de l'Église dans le tissu social de son temps, telle qu'une généalogie de la notion de scandale articulée à ses usages pratiques aurait permis de l'appréhender (à cet égard, le livre de Giacomo TodeschinI, Au pays des sans-nom : gens de mauvaise vie, personnes suspectes ou ordinaires du Moyen Âge à l'époque moderne, Nathalie Gailius [trad.] et Patrick Boucheron [préf.], Lagrasse, Verdier [Histoire], 2015, reste irremplaçable et unique en son genre, à la frontière d'une analyse serrée des constructions juridiques et d'une histoire politique et sociale du monde communal italien). En solide juriste, W. Druwé compile d'excellentes fiches par œuvres ou par auteurs, mais n'en pointe pas toujours les problématiques communes (un·e historien ne aurait sans doute construit son propos autrement, en partant d'un chapitre sur la définition ou les acceptions du scandale, pour ensuite aborder la question de ses conséquences, et enfin celle des modalités du gouvernement ecclésial auxquelles cette catégorie du droit donna lieu - comme la dispense qui est certes évoquée p. 38-39, mais uniquement sous l'angle de ce que Rufin en dit). Ce choix de présentation fait qu'à aucun moment, n'est surmontée la diversité des usages du terme et n'est tenue la promesse de sémantique historique faite au début l'auteur concluant que le scandale est « une notion à contenu variable » (p. 89) ou un « concept juridique très vague $»($ p. 91$)$.

L'auteur échappe heureusement à l'écueil d'une lecture sociologisante qui verrait dans le scandalum un état de fait immanent aux communautés dans lesquelles il prend sa source - à l'image du gossip et de la rumeur fantasmés par certains médiévistes sous l'influence de l'École anthropologique de Manchester - et montre à quel point il s'agit au contraire d'une catégorie juridique complexe, qui précède systématiquement le travail de qualification des juges. Certaines questions toutefois demeurent en suspens : que signifie, par ex., le passage, voire la substitution, dans certains textes, du pluriel scandala au singulier scandalum? C'est une question capitale à laquelle l'historiographie francophone n'a jusqu'ici pas répondu, et à laquelle l'auteur ne prête pas assez attention lorsqu'il traduit les deux termes par « conflits »- tout en précisant ensuite que « le scandale » au singulier peut être la source « scandales » au pluriel (p. 91). De même, quel déplacement s'opère lorsque la notion ne s'applique plus seulement aux clercs, mais aussi aux laïcs? Pour ma part, j'ai longtemps pensé que l'obsession des juristes pour le scandale était principalement liée à leur crainte des effets causés par le mauvais exemple donné par les clercs (autrement dit à l'hérésie et au schisme); et $\mathrm{W}$. Druwé a tout à fait raison de conclure que l'un des enjeux sous-jacents de cette catégorie du droit est « la protection de l'Église comme institution » (p. 93). Mais que circonscrit le scandale lorsqu'il désigne les effets de l'adultère? Dans le dernier chapitre, l'auteur évoque un cas saisissant, tiré de la Summa de penitentia de Raymond de Peñafort. Il est question de quelqu'un qui n'a rien à se reprocher devant Dieu, mais dont la réputation est mauvaise au point de « scandaliser» les autres. Ce cas tend à montrer que le scandale devint pour les juristes et les théologiens du XIII ${ }^{\mathrm{e}} \mathrm{s}$. l'opérateur leur permettant de penser l'articulation entre ce que Thomas d'Aquin appellera quelques années plus tard le « for de la conscience » et la réputation, ou pour le dire dans les termes de la modernité, entre l'individu et la société. W. Druwé ne s'y est donc pas trompé : s'il est bien un signifiant médiéval redevable d'une authentique « sémantique historique », c'est le scandalum.

Arnaud FossIER

UMR 7366 - LIR3S, Université de Bourgogne 